\title{
SUBSURFACE FAULT IMAGING USING CROSSHOLE TOMOGRAPHIC METHODS
}

\author{
L.F. IOANNIS
}

\begin{abstract}
Crosshole seismic tomography experiments were performed to image the subsurface structure between pairs of wells in three locations at Ano Liosia Municipality. Seismic caps were used as downhole sources and a 24channel geophone cable provided the downhole receivers. The crosshole data were processed with a SIRT traveltime transmission tomography package to produce a velocity model. The crosshole velocity section exhibits rapid lateral and vertical velocity changes that are not resolved by surface methods. The results indicate that crosshole tomography can potentially be used to image steeply dipping beds or complex structures, such as small faults or cavities.
\end{abstract}

KEY WORDS: cross-borehole tomography, traveltime inversion, high resolution imaging

\section{ПЕРІАНЧн}

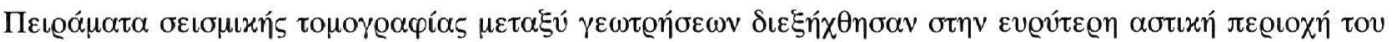

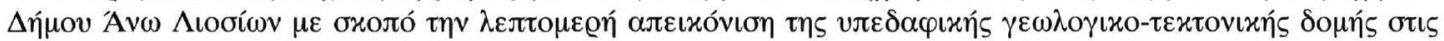

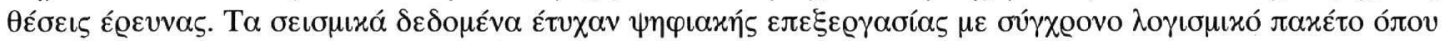

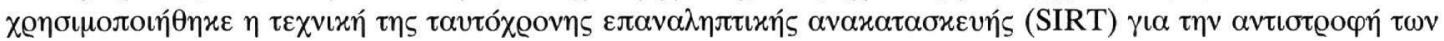

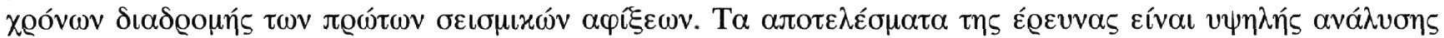

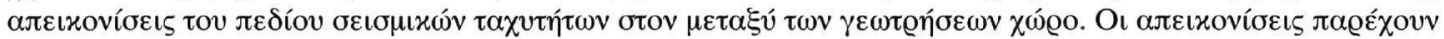

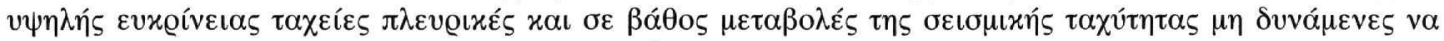

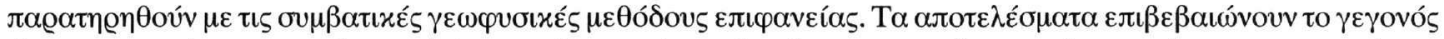

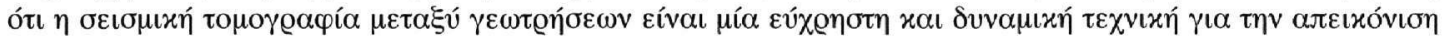

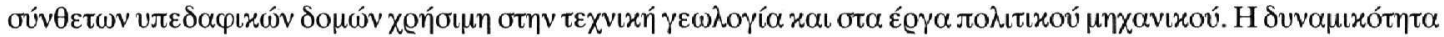

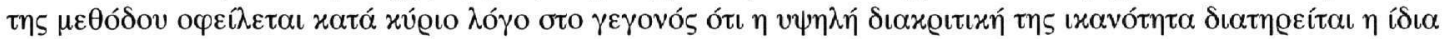

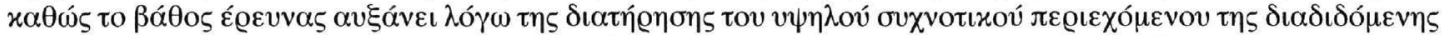

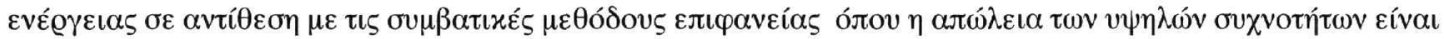

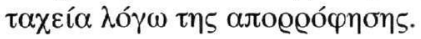

\section{INTRODUCTION}

In crosshole seismic tomography, seismic sources located in a well are shot into receivers located in a nearby well. The travel times of the first arrivals are used to produce a tomographic velocity cross-section of the subsurface between the two wells (Bregman et al., 1989; Calnan and Schuster, 1989; Lines and LaFehr, 1989; McMechan et al., 1987). Crosshole tomography is expected to provide better resolution than VSP or RVSP since most of the energy does not travel through the earth's highly attenuating near surface and the travel distances are shorter. In addition, the resolution of crosshole tomography is not depth limited since most of the energy travels between the wells.

\section{THE EXPERIMENTS}

The crosshole tomographic experiments were performed in three locations $\alpha \tau$ Ano Liosia Municipality (Figure 1) between pairs of properly cased wells 70 meters depth. In each location a $2 \mathrm{D}$ resistivity tomography section is running across the wells, imaging the subsurface structure of the greater lateral and in depth area.

The crosshole traveltime tomography experiment (Figure 2) comprises sources located in one well shot into receivers located in the other well (hole to hole).

1. Geophysics \& Geothermics Dpt., University of Athens, Panepistimiopolis Ilisia 15784, Greece.

E-mail: jlouis@geol.uoa.gr 


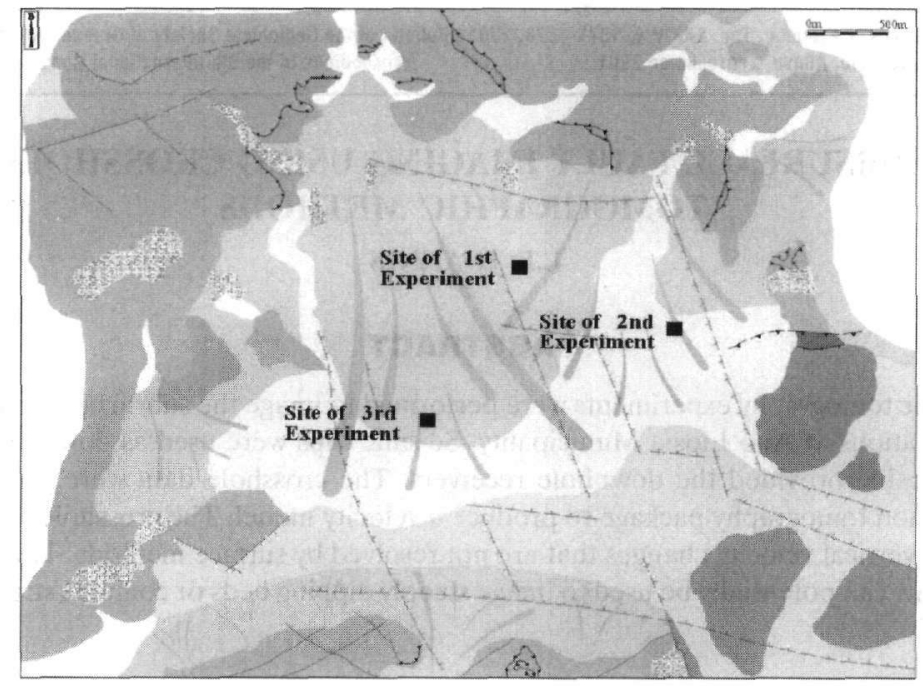

Figure 1. Location map with the sites of crosshole seismic tomography experiments.

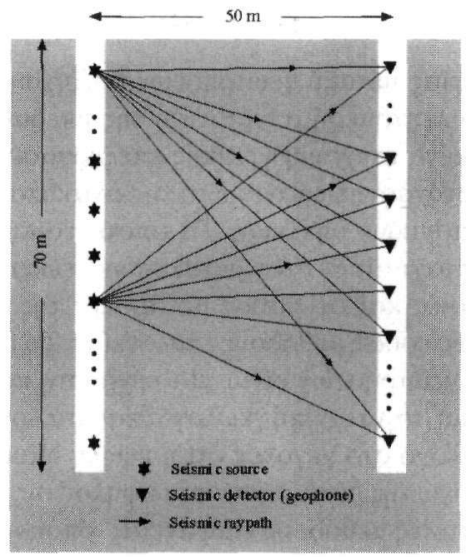

Figure 2. Schematic diagram of the crosshole tomography experiment.

A 24-channel geophone cable coupled with $14 \mathrm{~Hz}$ vertical component geophones served as the receiver. The space between receiver stations was 2.5 meters. The sources used in this experiment were seismic cap charges fired at 2.5 meters intervals from a depth of 70 meters to 5 meters. The seismic cap charge provided high quality data and did not damage the well. The seismic signals from all the stations were transmitted uphole simultaneously through tow leaders and recorded with a surface EG\&G 24 channel recording system. Only two geophone cable positions were required to cover the receiver well from depths 70 meters to 5 meters.

\section{DATA ANALYSIS AND PROCESSING}

Examples of crosshole seismic data collected at selected shot locations are displayed in Figures 3 and 4. Twenty-eight shots ranging from depths 70 meters to 5 meters in the source well at 2.5 meters intervals were recorded for each receiver station. 


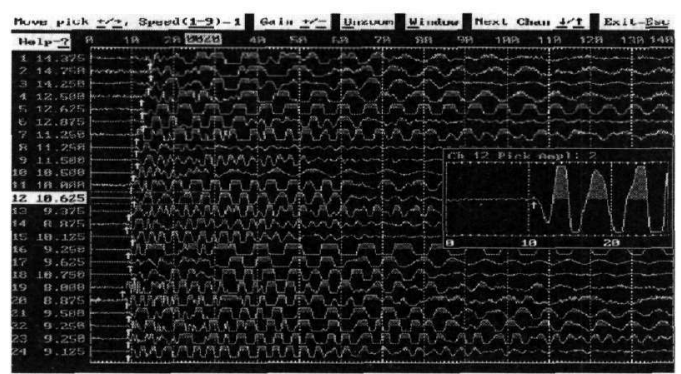

Figure 3. Seismic record from crosshole experiment in pair W1-W2.

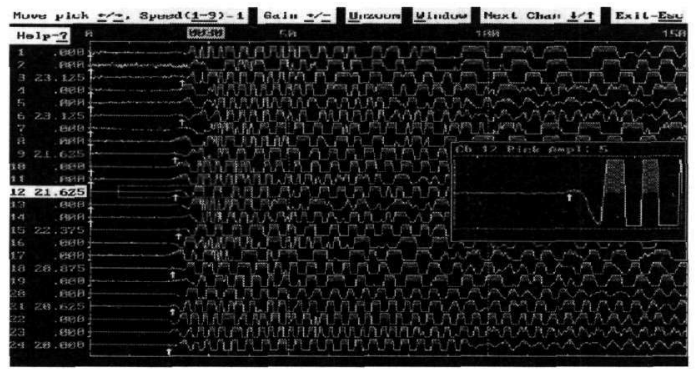

Figure 4. Seismic record from crosshole experiment in pair W6-W7.

Data in figures 3 and 4 were recorded by downhole geophones in wells W1 and W6 from the respective transmitting wells W2 and W7.

In crosshole transmission tomography, the first-arrival $\mathrm{P}$ waves are used to produce a velocity cross-section. Therefore, the precision of the travel time measurement is vital to the resolution of the final velocity crosssection. Since the $\mathrm{S} / \mathrm{N}$ ratio of the data is good an automatic picking program based in threshold technique was used to pick the first-arrival times. We were able to pick the first-arrival times precisely on at least 85 percent of the $1950(2 \times 750+450)$ total traces of the three-crosshole experiments. The precision of the picks is approximately $0.125 \mathrm{~ms}$.

Tomographic imaging is based on ray theory. The region bordered by the two boreholes is divided into rectangular cells or pixels, a contraction of "picture elements". The velocity of each cell is assumed to be constant and unknown.

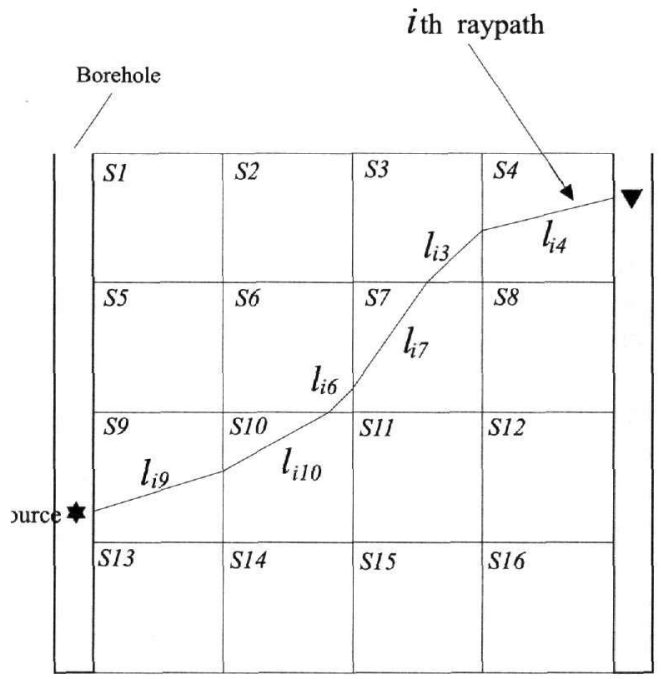

Figure 5. Schematic illustration of ray paths through a cell slowness model

(After J. Berryman, 1991). 
Referring to Figure 5, let $t_{i}$ be the first-arrival travel time for the $i$ th ray, $l_{i j}$ be the distance traveled across the $j$ th cell by the $i$ th ray, and $\mathbf{s}_{j}$ be the slowness of the $j$ th cell (slowness is the inverse of velocity). It is easy to see that

$$
t_{i}=\sum_{j=1}^{N} l_{i j} s_{j}
$$

Where $N$ is the total number of pixels. Note that for any given $i$, the ray path lengths $l_{i j}$ are zero for most cells $\boldsymbol{j}$, as a given ray path will in general intersect only a few of the cells in the model. We can rewrite equation (1) in matrix notation by defining the column vectors $\boldsymbol{s}$ and $\boldsymbol{t}$ and the matrix $\boldsymbol{M}$ as follows:

$$
\mathbf{S}=\left[\begin{array}{c}
s_{1} \\
s_{2} \\
\vdots \\
s_{n}
\end{array}\right]
$$

$$
\mathbf{t}=\left[\begin{array}{c}
t_{1} \\
t_{2} \\
\vdots \\
t_{n}
\end{array}\right]
$$$$
\mathbf{M}=\left[\begin{array}{cccc}
l_{11} & l_{12} & \cdots & l_{1 n} \\
l_{21} & l_{22} & \cdots & l_{2 n} \\
\vdots & \vdots & \ddots & \vdots \\
l_{m 1} & l_{m 2} & \cdots & l_{m n}
\end{array}\right]
$$

Equation (1) then becomes the basic equation of forward modeling for ray equation analysis:

$$
\mathbf{M s}=\mathbf{t}
$$

Given the travel times vector $\mathbf{t}$ and the raypaths matrix $\mathbf{M}$, we need to solve for the slowness vector $\mathbf{s}$. The number of rows of $\mathbf{M}$ equals the number of rays (first-arrival time picks). The number of columns of $\mathbf{M}$ equals the number of pixels (cells) in the image. For example, for the experiment between wells W1 and W2, we had

Number of pixels $=12 \times 25=300$

Number of rays $=750$

To invert the seismic data we used the CAT3D package developed by OGS within the frameworks of the European Union Project 3D Asymptotic Seismic Imaging. CAT3D is composed with programs for ray tracing, tomographic inversion, grid manipulation and display. The inversion algorithm is robust with respect to noise contaminating the data, and does not depend strongly on the a priori information as most conventional algorithms. It does not require particular data (as VSP's or cross-well geometries): good results are obtained generally also by conventional surface data alone. The Input/Output formats available allow the exchange of data and models with all de facto standards currently adopted (SEG-Y, Landmark, Geoquest, ASCII, etc.). The various parts exchange various information, as the model interfaces, pixel geometry, 2D or 3D velocity field, sources and receivers locations, ray paths, travel times and, also, set of parameters to guide the forward and inverse modeling.

The iterative method SIRT (Simultaneous Iterative Reconstruction Technique) was used for the inversion. The algorithm has two main components: Tracing the rays to obtain $\mathbf{M}$ (raypaths) and solving for the $\mathbf{s}$ (SIRT). All this is done in a SUN spark workstation. A "check board" test was performed leading to a pixel size 3 meters vertically and 4.16 meters horizontally maximizing in that way the recovery efficiency of the model. The first iteration was done with an initial guess of constant velocity of $3000 \mathrm{~m} / \mathrm{s}$. The regularized SIRT was run through 5 iterations and the obtained velocity field was used again as an initial velocity model to obtain the final velocity field after 10 more iterations.

Figure 6 shows the ray coverage for the investigated area between wells W1 and W2. The measure of ray coverage or ray density is a popular indicator to estimate the inversion reliability. This indicator is measured here as the number of rays crossing each pixel. Although it can be computed very easily, it is considered rather a poor indicator of the local reliability, because it does not distinguish linearly dependant from independent rays. Singular value analysis and null space energy indicators are used today alongside with ray density to estimate the reliability of the solution.

Figure 7 shows the final velocity field (model) obtained from the inversion of first-arrival times. The major events in the velocity-depth section are identified as follows:

1. The surface layer with a dipping interface and lateral velocity variations $(1300-2300 \mathrm{~m} / \mathrm{s})$, which is attrib- 
uted to the conglomerates formation.

2. The high velocity $(4000 \mathrm{~m} / \mathrm{s})$ deeper layer attributed to the limestone bedrock.

3. An intermediate velocity zone $(2400 \mathrm{~m} / \mathrm{s})$ intersecting the high velocity $(4000 \mathrm{~m} / \mathrm{s})$ rock mass in NE-SW direction, which is attributed to a main fault $F$.

4. Two intermediate velocity zones crossing the main fault $\mathrm{F}$ attributed to respective minor faults.

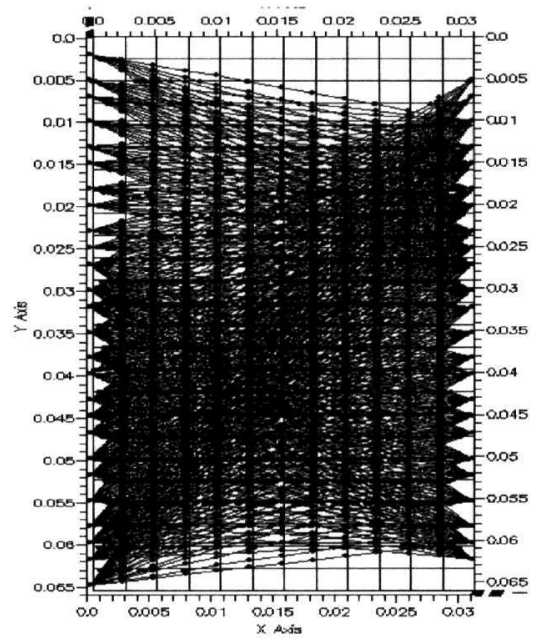

Figure 6. Ray coverage of the area between wells W1 and $W 2$.

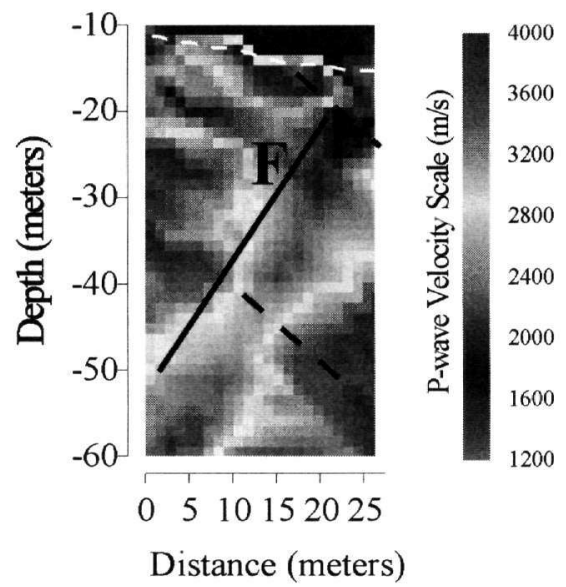

Figure 7. P-wave velocity image of the area between wells W1 and W2.

The upper part of Figure 8 shows a $2 \mathrm{D}$ resistivity tomography section running in the area of the first experiment. It images the lateral and vertical variations of the subsurface structure in the greater area. The lower part of the same figure demonstrates and compares resistivity and tomographic seismic images of the same portion of the subsurface between the wells. The main fault F, running in NE-SW direction, delineated from seismic tomography image is also well outlined in the $2 \mathrm{D}$ resistivity model.

The resistivity-depth image does not show any other indication such as the bottom interface of the surface layer or the minor fault zone near the surface. On the contrary crosshole seismic tomography it appears to be capable of distinguishing lateral velocity changes than the $2 \mathrm{D}$ resistivity method. This can provide high-resolution subsurface images that are of valuable information for engineering geologists and civil engineers.

Unfortunately, coverage near the top and bottom of the wells is poor (Figure 6). In order to effectively delineate the investigated area combined seismic techniques should be employed. The lack of ray coverage in 
the upper part area between the wells (Figure 6) can be overcome by combining crosshole seismic with Vertical Seismic Profiling (VSP) and/or Reverse Vertical Seismic Profiling (RVSP) techniques. Concerning the lack of information for the lower part area between the wells (Figure 6), the wells need to be drilled considerably deeper than the zone of interest.

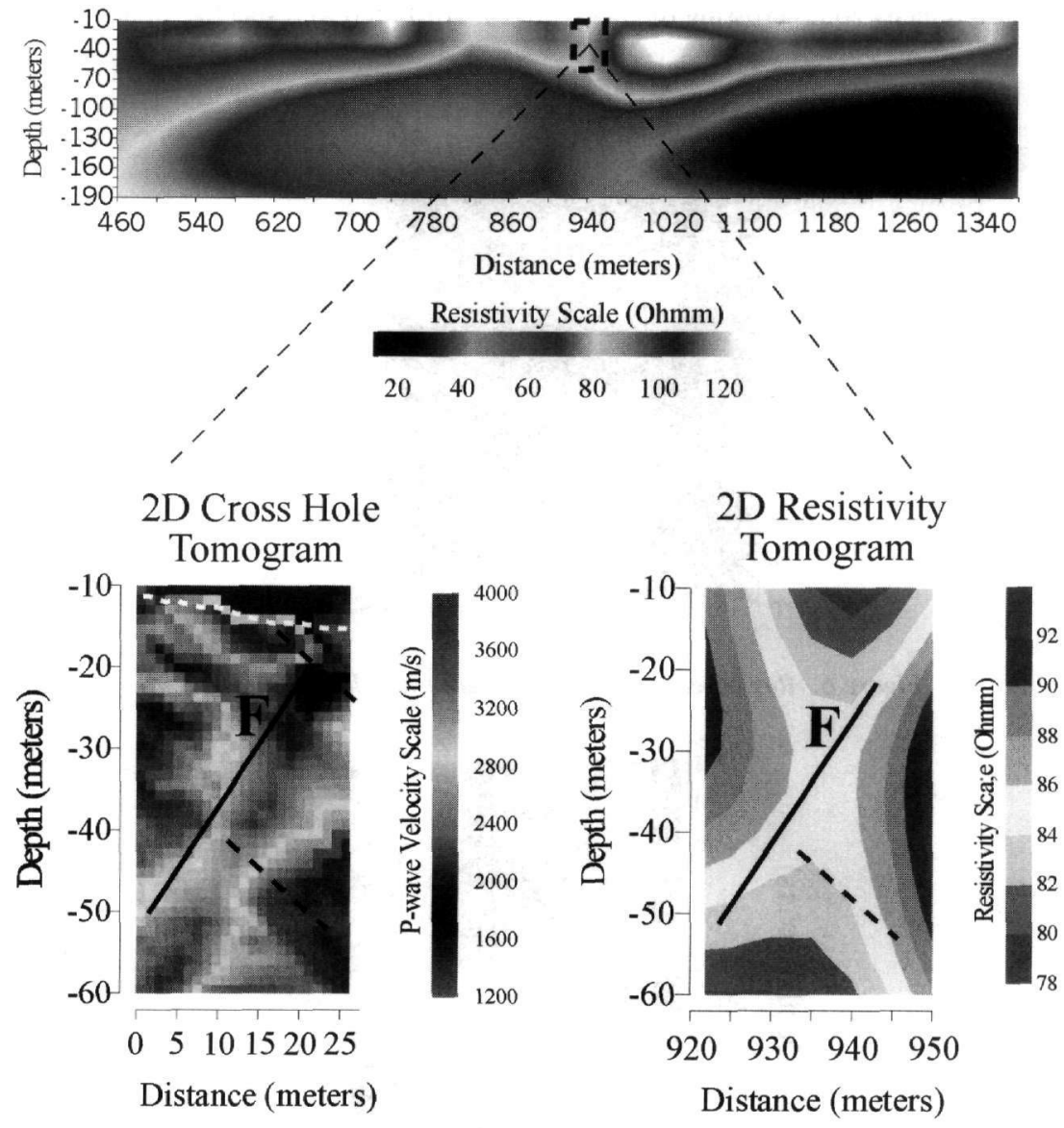

Figure 8. The upper part shows a $2 D$ resistivity section running in the area of the first experiment. It images the lateral and vertical variations of the subsurface structure in the greater area. The lower part compares resistivity and seismic images of the same portion of the subsurface.

The upper part of figure 9 shows a $2 \mathrm{D}$ resistivity section in the area of the second experiment. It images the lateral and vertical variations of the subsurface structure in the greater area. The final velocity field (model) obtained from the inversion of first-arrival times, recorded in the experiment between pair W3-W4, is shown in the lower part (b) of the same figure. The major events identified in the velocity-depth section are as follows:

1. The surface layer of variable depth and lateral variations of seismic velocity $(1300-3000 \mathrm{~m} / \mathrm{s})$, which is attributed to the conglomerates formation.

2. The intermediate layer with low to intermediate velocities $(1400-2500 \mathrm{~m} / \mathrm{s})$.

3. The high velocity $(3500 \mathrm{~m} / \mathrm{s})$ basement layer. The major lateral velocity variation $(2500$ to $3500 \mathrm{~m} / \mathrm{s})$ observed in the middle of the velocity model is attributed to a main fault zone F striking NE-SW.

4. The intermediate velocity zone $(2400 \mathrm{~m} / \mathrm{s})$ observed in the lower right part of the figure, intersecting the high velocity basement rock, is attributed to a minor fault zone. 


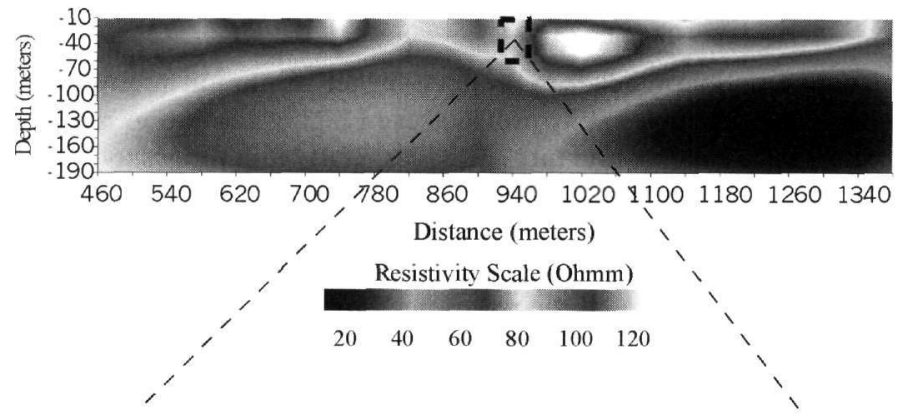

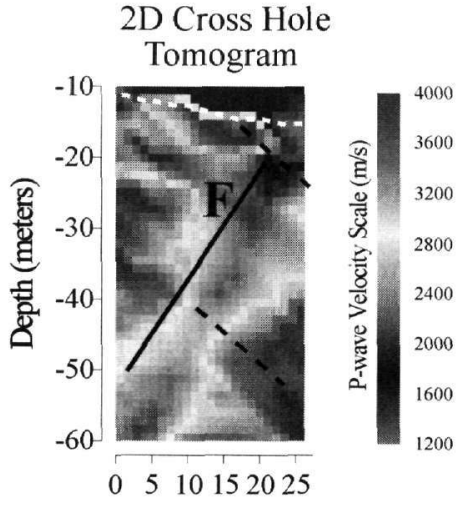

Distance (meters)

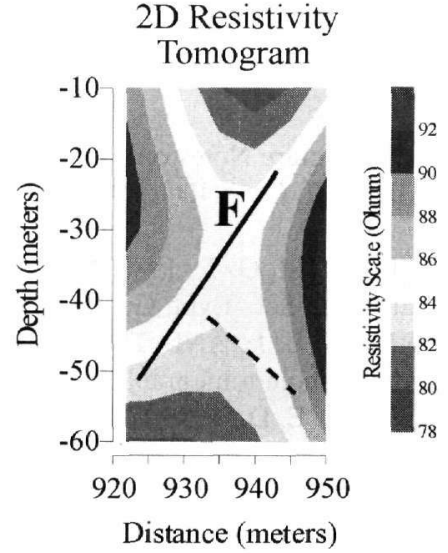

Figure 9. A 2D resistivity tomography section, running in the area of the second experiment, is shown in the upper part. It images the lateral and vertical variations of the subsurface structure in the greater area. The lower part compares resistivity (a) and seismic (b) images of the same portion of the subsurface.

Another $2 \mathrm{D}$ resistivity section, running in the area of the third experiment, is shown in the upper part of figure 10. It images the lateral and vertical variations of the subsurface structure in the greater area. The final velocity field (model) obtained from the inversion of first-arrival times recorded in the experiment between pair W5-W6 is shown in the lower part (b) of the same figure.

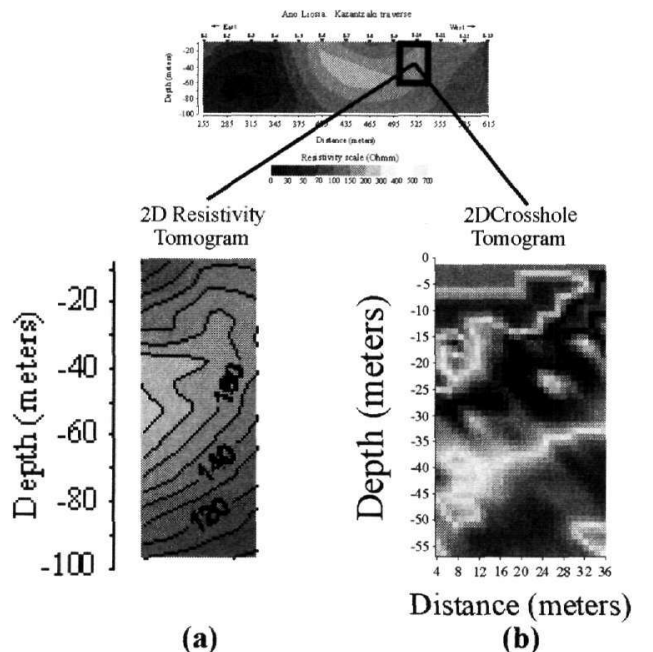

Figure 10. 2D resistivity tomogram from the area of the third experiment (upper part). The lower part compares resistivity (a) and seismic (b) images of the same portion of the subsurface. 


\section{CONCLUSIONS}

Crosshole seismic tomography experiments can produce higher resolution subsurface images than surface seismic or resisitivity data. Crosshole tomography provides even higher resolution cross-sections than surface methods and potentially can resolve smaller structures. Cavities, faults with throws too small to be resolved with surface seismic data are examples of exploration targets that crosshole seismic tomography may adequately resolve. This, in large part, is due to less attenuation of high frequencies in the crosshole data.

Unlike RVSP and surface seismic where all the waves need to travel to the surface, most of the crosshole energy travels only between the wells. Therefore, the subsurface images produced by crosshole tomography will maintain the same high resolution as the depth increases, while the images produced by RSVP or surface seismic will lose their resolution. In addition, crosshole tomography can distinguish both lateral and vertical velocity changes, which can provide valuable information about rock properties.

\section{REFERENCES}

BERRYMAN, J. G., 1991, Lecture Notes on Nonlinear Inversion and Tomography: LLNL, Livermore, CA.

BREGMAN, N. D., BAILEY, R. C., AND CHAPMAN, C. H., 1989, Crosshole seismic tomography: Geophysics, 54, 200-215

CALNAN, C., AND SCHUSTER, G. T., 1989, Reflection and transmission cross-well tomography: Presented at the $59^{\text {th }}$ Ann. Internat. Mtg., Soc. Expl. Geophys., Expanded Abstracts, 908-911.

CHEN, S. T., ZIMMERMAN, L. J., AND TUGNAIT, J. K., 1990, Subsurface imaging using reversed vertical seismic profiling and crosshole tomographic methods: Geophysics, 55, 1478-1487.

LINES, L. R., AND LAFEHR, E. D., 1989, Tomographic modeling of a cross-borehole data set: Geophysics, 54, 1249-1257.

McMECHAN, G. A., HARRIS, J. M., AND ANDESRON, L. M., 1987, Cross-hole tomography for strongly variable media with applications to scale model data: Bull. Seis. Soc. Am., 77, 1945-1960. 